PROCEEDINGS OF THE

AMERICAN MATHEMATICAL SOCIETY

Volume 137, Number 9, September 2009, Pages 2869-2877

S 0002-9939(09)09880-3

Article electronically published on April 17, 2009

\title{
ON NON-UNIFORM RAMANUJAN COMPLEXES
}

\author{
BETH SAMUELS
}

(Communicated by Wen-Ching Winnie Li)

\section{INTRODUCTION}

Ramanujan graphs were defined in [LPS as finite connected $k$-regular nondirected graphs for which

$$
\operatorname{spec} A_{X} \subseteq\{ \pm k\} \cup[-2 \sqrt{k-1}, 2 \sqrt{k-1}],
$$

where $A_{X}$ is the adjacency matrix of the graph $X$. Such graphs were constructed there as quotients of the Bruhat-Tits tree associated to $G=\operatorname{PGL}_{2}\left(\mathbb{Q}_{p}\right)$, modulo congruence subgroups of uniform (i.e. cocompact) lattices of $G$, where $\mathbb{Q}_{p}$ is the field of the $p$-adic integers. More examples were given by Morgenstern M1 by replacing $\mathbb{Q}_{p}$ by a local field $F$ of characteristic $p>0$, such as $\mathbb{F}_{q}((t))$. One significant difference is that unlike $\mathrm{PGL}_{2}\left(\mathbb{Q}_{p}\right), \mathrm{PGL}_{2}\left(\mathbb{F}_{q}((t))\right)$ has a non-uniform lattice $\Delta=$ $\mathrm{PGL}_{2}\left(\mathbb{F}_{q}[1 / t]\right)$, which is of finite covolume but not cocompact. For congruence subgroups of $\Delta$, the quotient graphs are infinite, but the edges and vertices come with weights, so that the total weight is finite. Morgenstern [M2 went ahead to define Ramanujan diagrams as such weighted objects satisfying Equation (1.1), where this time $A_{X}$ is the 'weighted adjacency matrix' (see Section 2 for more details). He constructed explicit examples and even showed a surprising application of them to the theory of networks M3.

Recently, the concept of Ramanujan graphs was generalized to higher dimensional complexes [Li, LSV1, Sa] (see Remark 4.5], (2)) by replacing $\mathrm{PGL}_{2}(F)$ by $\mathrm{PGL}_{d}(F)$ for $d>2$. This time one requires bounds on the simultaneous spectrum of $d-1$ 'Hecke operators', which are 'colored adjacency matrices'; see Section 3 below.

Such complexes were constructed (see [LSV2, Sa]) as quotients of the Bruhat-Tits building $\mathcal{B}_{d}$ associated with $\mathrm{PGL}_{d}(F)$, modulo congruence subgroups of uniform lattices of $\mathrm{PGL}_{d}(F)$, assuming the Jacquet-Langlands correspondence in positive characteristic.

The current work started from an attempt to construct non-uniform Ramanujan quotients of $\mathcal{B}_{d}$, generalizing the finite (uniform) Ramanujan complexes of LSV2, just like Morgenstern extended the theory of (finite) Ramanujan graphs to (nonuniform) Ramanujan diagrams. Quite surprisingly, we prove the opposite:

Received by the editors April 7, 2008, and, in revised form, December 1, 2008.

2000 Mathematics Subject Classification. Primary 11F70.

Posthumous, based on Chapter 3 of the author's Ph.D. thesis. Prepared for publication by Alex Lubotzky and Uzi Vishne.

(C)2009 American Mathematical Society Reverts to public domain 28 years from publication 
Theorem 1.1. Let $G=\mathrm{PGL}_{d}\left(\mathbb{F}_{q}((t))\right), \Delta=\mathrm{PGL}_{d}\left(\mathbb{F}_{q}[1 / t]\right)$, and $\mathcal{B}_{d}$ be as before, where $d>2$. Then, for any finite index subgroup $\Gamma$ of $\Delta, \Gamma \backslash \mathcal{B}_{d}$ is not Ramanujan.

We refer the reader to Section 3 for details.

In retrospect, this theorem is not that surprising. The Ramanujan conjecture (proved for PGL 2 over $\mathbb{Q}$ by Deligne and in positive characteristic by Drinfeld, and for $\mathrm{PGL}_{d}(d>2)$ in positive characteristic by Lafforgue [La] gives bounds on the cuspidal spectrum. But this is not the complete story: the other parts of the spectrum are easier to understand, but they do not satisfy the same bounds as the cuspidal spectrum, at least for $d>2$. See Section 4 for more on this.

While from the point of view of representation theory there are significant differences between the cuspidal spectrum and the other parts of the spectrum, the combinatorial meaning of the distinction is not clear. A better combinatorial characterization of the various parts of the spectrum may lead to an interesting theory of non-uniform weakly Ramanujan complexes in spite of our theorem (see Section 5).

The paper is organized as follows. In Section 2 we review the 1-dimensional case - the Ramanujan graphs and the Ramanujan diagrams in their combinatorial and representation-theoretic forms. In Section 3 we extend these definitions to the higher-dimensional case, while in Section 4 we show that actually the natural candidates for non-uniform Ramanujan complexes are not so. In Section 5 we discuss some other potential examples and the property of being weakly Ramanujan.

This work, published posthumously, is based on Chapter 3 of the author's thesis $[\mathrm{S}$. Thanks are due to Jonathan Rogawski and Erez Lapid for their help in the preparation of this paper.

\section{RAMANUJAN GRAPHS AND DIAGRAMS}

Let $X=(V, E)$ be a finite non-oriented graph; i.e. $V$ is a set of vertices and $E$ a set of edges between the vertices $V$, which we view as a (multi)set of pairs of elements of $V$.

Let $\mathrm{L}^{2}(X)$ be the space of complex-valued functions on $V$, and let $A_{X}: \mathrm{L}^{2}(X) \rightarrow$ $\mathrm{L}^{2}(X)$ denote the operator defined by $\left(A_{X} f\right)(v)=\sum_{(v, u) \in E} f(u)$.

Assume $X$ is $k$-regular for some integer $k$; i.e. for every $v \in V$, the number of edges (multiplicities counted) containing $v$ is $k$. Then the spectrum of $X$ is contained in the real segment $[-k, k]$. The endpoint $k$ is an eigenvalue of $A_{X}$ with the constant functions being the eigenvectors; it is an eigenvalue of multiplicity 1 iff $X$ is connected. On the other hand, $-k$ is an eigenvalue iff $X$ is bipartite.

Note that the adjacency operator $A$ acts also on $\mathrm{L}^{2}\left(T_{k}\right)$, where $T_{k}$ is the infinite $k$-regular tree, which is the universal cover of the finite $k$-regular graph $X$. The spectrum of $A$ on $\mathrm{L}^{2}\left(T_{k}\right)$ is $[-2 \sqrt{k-1}, 2 \sqrt{k-1}]$ (notice that the constant function is not in $\left.\mathrm{L}^{2}\left(T_{k}\right)\right)$.

Definition 2.1. A connected $k$-regular graph is called a Ramanujan graph if

$$
\operatorname{spec}\left(A_{X}\right) \subseteq\{ \pm k\} \cup[-2 \sqrt{k-1}, 2 \sqrt{k-1}] .
$$

Thus, the finite graph is Ramanujan iff all its eigenvalues are either trivial (i.e. $\pm k$ ) or in the spectrum of the universal cover (see $[\mathrm{Lu}]$ for more on that). By the Alon-Boppana theorem, this is asymptotically the smallest possible interval containing the spectrum (see [LPS] and [Lu]).

We say that $\Gamma$ is a congruence lattice in $\mathrm{PGL}_{d}(F)$ if $\Gamma$ is a congruence subgroup in some form $G^{\prime}$ of $\mathrm{PGL}_{d}(F)$. Specifically in the case of $\mathrm{PGL}_{2}$, every such form 
is the group of invertible elements in a quaternion algebra defined over a global subfield of $F$, modulo the center.

The following result presents examples of Ramanujan graphs. See [Lu, Chapter 7] and the references therein for more explanations.

Theorem 2.2. Let $F$ be a non-Archimedean local field with $q=|\bar{F}|$ the cardinality of the residue field, $G=\mathrm{PGL}_{2}(F)$, and $T=T_{q+1}$ the Bruhat-Tits tree associated with $G$. Then, for every congruence uniform lattice $\Gamma$ of $\mathrm{PGL}_{2}(F)$, the quotient $\Gamma \backslash T$ is a $(q+1)$-regular Ramanujan graph.

In [M2], Morgenstern introduced the notion of Ramanujan diagrams in order to study $\Gamma \backslash T$, when this time $\Gamma$ is a non-uniform lattice of $G$. More precisely:

Definition 2.3. A diagram is a triple $D=(V, E, \omega)$, where $(V, E)$ is a connected undirected graph, finite or countable, and $\omega: V \cup E \rightarrow[0,1]$ is the weight function.

Call $\omega(e) / \omega(u)$ the entering degree of $e=(u, v)$ to $u$, and for a vertex $u$, in- $\operatorname{deg}(u)=\sum_{(u, v) \in E} \frac{\omega(u, v)}{\omega(u)}$. We say that $D$ is $k$-regular if, for every $u \in V$, $\operatorname{in}-\operatorname{deg}(u)=k$.

The measure on $D$ is defined by $\mu(S)=\sum_{u \in S} \omega(u)$ for any $S \subseteq V$, and we assume that $\mu(V)<\infty$.

An inner product is defined on the space of functions $V \rightarrow \mathbb{C}$ by

$$
\langle f, g\rangle=\int_{D} f \bar{g} d \mu=\sum_{u \in V} \omega(u) f(u) \overline{g(u)} .
$$

The norm $\|f\|$ is defined as usual, and we obtain the space $\mathrm{L}^{2}(V)$. Let $A=A_{D}$ be the adjacency operator on $\mathrm{L}^{2}(V)$, defined by

$$
\left(A_{D} f\right)(u)=\sum_{(u, v) \in E} \frac{\omega(u, v)}{\omega(u)} f(v) .
$$

It is worth noting that the measure and the norm only depend on weights of vertices, while $A_{D}$ is tuned further by the weight of edges.

It is not difficult to see that $A$ is a bounded operator, and when $D$ is $k$-regular, we have that $\operatorname{spec}(A) \subseteq[-k, k]$.

Definition 2.4. A $k$-regular diagram $D$ is a Ramanujan diagram if

$$
\operatorname{spec}\left(A_{D}\right) \subseteq\{ \pm k\} \cup[-2 \sqrt{k-1}, 2 \sqrt{k-1}] .
$$

Note that if $D$ is infinite, the spectrum has a continuous part in addition to its discrete part. Finally, if $F, G$ and $T=G / K$ are as in Theorem 2.2 , and $\Gamma \leq G$ is a (possibly non-uniform) lattice, then $\Gamma \backslash T$ becomes a diagram when the weights are defined by $\omega(u)=|\{\gamma \in \Gamma: \gamma u=u\}|^{-1}$ and $\omega(e)=|\{\gamma \in \Gamma: \gamma e=e\}|^{-1}$ for any vertex $u$ and edge $e$. Under this definition, the Laplacian $A$ of $T$ induces the weighted $A_{D}$ on the quotient.

Morgenstern then shows:

Theorem 2.5. Let $F, G$ and $T$ be as in Theorem 2.2 , and $\Delta=\mathrm{PGL}_{2}\left(\mathbb{F}_{q}[1 / t]\right)$. If $\Gamma$ is a congruence subgroup of $\Delta$, then $\Gamma \backslash T$ is a Ramanujan diagram.

Note that such a non-uniform lattice $\Delta$ in $G$ exists only when $F$ is of positive characteristic.

The proofs of Theorems 2.2 and 2.5 are quite similar. Both are based on the following representation-theoretic reformulation: 
Proposition 2.6. In the notation above, if $\Gamma$ is a lattice in $G$ (whether uniform or not), then $\Gamma \backslash X$ is Ramanujan if and only if all the infinite-dimensional spherical irreducible $G$-representations, which are weakly contained in $\mathrm{L}^{2}(\Gamma \backslash G)$, are tempered (which in the case of $G=\mathrm{PGL}_{2}(F)$ means not from the complementary series).

Again, we refer to $[\mathrm{Lu}$ and the appendix there for explanations of the notions used here. Now, the Ramanujan conjecture (or, more precisely, the RamanujanPeterson conjecture), which was proved for $\mathrm{PGL}_{2}$ by Deligne for $F=\mathbb{Q}_{p}$ and by Drinfeld for $\operatorname{char}(F)>0$, indeed ensures that the representation-theoretic condition of Proposition 2.6 is satisfied, and thus Theorems 2.2 and 2.5 follow.

\section{Ramanujan COMPleXes}

The work of Lafforgue La, who proved higher-dimensional analogs of the Ramanujan conjecture for $\mathrm{PGL}_{d}(F)$, where $F$ is of positive characteristic, opened the door for higher-dimensional analogs of Ramanujan graphs, the so-called Ramanujan complexes [Li], LSV1. We recall here briefly the definition, sending the reader to [LSV1, Section 2] for more details.

Let $d \geq 2$ and $G=\mathrm{PGL}_{d}(F)$. The affine building $\mathcal{B}=\mathcal{B}_{d}(F)$ associated with $G$ is a $(d-1)$-dimensional simplicial complex, which can be constructed as follows. Let $\mathcal{O}$ denote the valuation ring in $F$. The set of vertices $\mathcal{B}^{0}$ in $\mathcal{B}$ is composed of the equivalence classes of full $\mathcal{O}$-submodules of $F^{d}$, where two modules are equivalent if they are obtained from each other by multiplication by a constant. The natural action of $\mathrm{GL}_{d}(F)$ on bases of $F^{d}$ defines an action of $G$ on $\mathcal{B}^{0}$, which can be identified with $G / K$ for $K=\mathrm{PGL}_{d}(\mathcal{O})$, as in the case $d=2$.

Two classes $\alpha$ and $\alpha^{\prime}$ are connected by an edge if there are representatives $M \in \alpha$ and $M^{\prime} \in \alpha^{\prime}$ such that $\pi M \subset M^{\prime} \subset M$, where $\pi \in \mathcal{O}$ is a (fixed) uniformizer. Higher-dimensional cells are formed by chains $\pi M_{0} \subset M_{\ell-1} \subset \cdots \subset M_{1} \subset M_{0}$. Thus, maximal cells in which the vertex $[M]$ participates correspond to maximal chains inside the $d$-dimensional vector space $M / \pi M$.

The $d$-coloring (sometimes called 'labelling') of the vertices is obtained by comparing vertices to the fixed submodule $\mathcal{O}^{d}$ : by definition, the color $\varrho([M])$ of the vertex $[M]$ is the logarithmic index $\log _{q}\left[M: \pi^{i} \mathcal{O}^{d}\right](\bmod d)$ for $i$ large enough, where $q=|\mathcal{O} / \pi \mathcal{O}|$. Under this coloring, every maximal cell contains a unique vertex of each color. The action of $G$ on $\mathcal{B}^{0}$ induces an action on the complex $\mathcal{B}$, which preserves the difference of colors of vertices, modulo $d$.

Let $A_{i}, i=1, \ldots, d-1$, be the $d-1$ colored adjacency operators acting on $\mathrm{L}^{2}\left(\mathcal{B}^{0}\right)$. (The $A_{i}$ are also called the Hecke operators.) These are defined by summing over colored edges: $\left(A_{i} f\right)(u)=\sum_{(u, v) \in \mathcal{B}^{1}, \varrho(u)-\varrho(v)=i} f(v)$. As explained there, these operators commute with each other and are bounded and normal. Their simultaneous spectrum, denoted $\mathcal{S}_{d}$, is a subset of $\mathbb{C}^{d-1}$ which is explicitly described in [LSV1, Subsection 2.3]. Similarly to the case $d=2$, where $\pm k$ are the trivial eigenvalues, there is a set of $d$ 'trivial eigenvalues' in $\mathbb{C}^{d-1}$.

If $\Gamma$ is a cocompact lattice in $G$, then the action of $A_{1}, \ldots, A_{d-1}$ on $\mathrm{L}^{2}(\mathcal{B})$ induces an action also on $\mathrm{L}^{2}(\Gamma \backslash \mathcal{B})$. We have:

Definition 3.1. The finite simplicial complex $\Gamma \backslash \mathcal{B}$ is called a Ramanujan complex if the eigenvalues of every non-trivial simultaneous eigenfunction $f \in \mathrm{L}^{2}(\Gamma \backslash \mathcal{B})$, $A_{i} f=\lambda_{i} f$, satisfy $\left(\lambda_{1}, \ldots, \lambda_{d-1}\right) \in \mathcal{S}_{d}$.

Similarly to Proposition 2.6, we have: 
Proposition 3.2 ([LSV1, Prop. 1.5]). Let $\Gamma$ be a cocompact lattice in $\mathrm{PGL}_{d}(F)$. Then $\Gamma \backslash \mathcal{B}$ is a Ramanujan complex iff every irreducible spherical infinite dimensional $G$-representation of $\mathrm{L}^{2}(\Gamma / G)$ is tempered.

Combining this with results from representation theory and assuming the Jacquet-Langlands correspondence in positive characteristic, we proved:

Theorem 3.3 ([LSV1, Theorems 1.2, 1.3]). Let $F$ be a local field of positive characteristic and $G=\mathrm{PGL}_{d}(F)$. Let $\Gamma$ be a congruence subgroup in an arithmetic uniform lattice of inner type in $G$.

Then: when $d$ is prime, $\Gamma \backslash \mathcal{B}$ is a Ramanujan complex. When $d$ is not a prime, $\Gamma \backslash \mathcal{B}$ is Ramanujan infinitely often and non-Ramanujan infinitely often.

See [LSV1] for more details. As for arithmetic lattices of outer type (which exist when $d>2$ ), we do not know if their respective quotients are Ramanujan; see [LSV1, Section 6].

\section{NON-UNIFORM COMPLEXES}

Fix $d \geq 2$, and let $F$ be a non-Archimedean local field, $G=\operatorname{PGL}_{d}(F)$, and $\mathcal{B}=\mathcal{B}_{d}$ the associated affine building, on which $G$ acts. Let $K$ denote the stabilizer of a point in the action of $G$ on $\mathcal{B}^{0}$; e.g. $K=\operatorname{PGL}_{d}(\mathcal{O})$. Then $K$ is a maximal compact subgroup of $G$, and $\mathcal{B}^{0}$ can be identified with $G / K$.

Let $\Gamma \leq G$ be a non-uniform lattice and consider the quotient $\Gamma \backslash G / K=\Gamma \backslash \mathcal{B}$, which is an infinite complex. One may adjoin weight functions on cells in the complex, just as in the case of diagrams. As in Section 2, the weight $\omega(p)$ of a vertex $p$ is defined as $|\{\gamma \in \Gamma: \gamma p=p\}|^{-1}$, and likewise we have $\omega(s)=|\{\gamma \in \Gamma: \gamma s=s\}|^{-1}$ for any cell $s$. Then $\omega$ is a well-defined weight function on $\Gamma \backslash \mathcal{B}$, as in Definition 2.3. Since $\Gamma \backslash G$ has finite volume, it follows that the total weight of $\Gamma \backslash \mathcal{B}^{0}$ is finite.

Recall the definition of the inner product on $\mathrm{L}^{2}\left(\Gamma \backslash \mathcal{B}^{0}\right)$ from (2.2). The colored operators are defined on $\Gamma \backslash G$ similarly to (2.3), by setting $\left(A_{i} f\right)(u)=$ $\sum_{(u, v) \in \mathcal{B}^{1}, \varrho(u)-\varrho(v)=i} \frac{\omega(u, v)}{\omega(u)} f(v)$. We remark that if $\Gamma$ is color-preserving, or if $d$ is odd, then every element $\gamma \in \Gamma$ which stabilizes an edge will certainly stabilize the corresponding vertices, so then $\frac{\omega(u, v)}{\omega(u)}$ is the index of the stabilizer of $(u, v)$ in the stabilizer of $u$, both being subgroups of $\Gamma$.

Remark 4.1. The adjacency operators are adjoint in pairs, namely $\left\langle A_{i} f, g\right\rangle=$ $\left\langle f, A_{d-i} g\right\rangle$ for every $f, g \in \mathrm{L}^{2}\left(\mathcal{B}^{0}\right)$.

Proof. For a vertex $u \in \mathcal{B}^{0}$, we denote by $\sum_{(u, v) \in \mathcal{B}^{1}}$ the sum over edges of color $\varrho(u)-\varrho(v)=i$. Then

$$
\begin{aligned}
\left\langle A_{i} f, g\right\rangle & =\sum_{u \in \mathcal{B}^{0}} \omega(u)\left(A_{i} f\right)(u) \overline{g(u)} \\
& =\sum_{u \in \mathcal{B}^{0}} \omega(u) \sum_{(u, v) \epsilon_{i} \mathcal{B}^{1}} \frac{\omega(u, v)}{\omega(u)} f(v) \overline{g(u)} \\
& =\sum_{u \in \mathcal{B}^{0}} \sum_{(u, v) \in_{i} \mathcal{B}^{1}} \omega(u, v) f(v) \overline{g(u)} \\
& =\sum_{u, v:(u, v) \in_{i} \mathcal{B}^{1}} \omega(u, v) f(v) \overline{g(u)}
\end{aligned}
$$




$$
\begin{aligned}
& =\sum_{u, v:(v, u) \in_{d-i} \mathcal{B}^{1}} \omega(v, u) f(v) \overline{g(u)} \\
& =\sum_{u, v:(v, u) \epsilon_{d-i} \mathcal{B}^{1}} \omega(v) f(v) \frac{\omega(v, u)}{\omega(v)} \overline{g(u)} \\
& =\sum_{v \in \mathcal{B}^{0}} \omega(v) f(v) \sum_{u, v:(v, u) \epsilon_{d-i} \mathcal{B}^{1}} \frac{\omega(v, u)}{\omega(v)} \overline{g(u)} \\
& =\sum_{v \in \mathcal{B}^{0}} f(v) \omega(v) \overline{\left(A_{d-i} g\right)(v)}=\left\langle f, A_{d-i} g\right\rangle .
\end{aligned}
$$

The operators $A_{1}, \ldots, A_{d-1}$ are normal and commute. Consequently, they have a simultaneous spectrum, defined as the vectors $\left(\lambda_{1}, \ldots, \lambda_{d-1}\right)$ in $\mathbb{C}^{d-1}$ for which there exists a sequence of unit vectors $x_{n} \in \mathrm{L}^{2}\left(\Gamma \backslash \mathcal{B}^{0}\right)$ such that $\lim _{n \rightarrow \infty}\left(A_{i} x_{n}-\right.$ $\left.\lambda_{i} x_{n}\right)=0$ for $i=1, \ldots, d-1$. As in the uniform case, there are $d$ one-dimensional spherical representations whose $K$-fixed vectors are considered trivial eigenvectors in $\mathrm{L}^{2}\left(\Gamma \backslash \mathcal{B}^{0}\right)$. These do not appear in $\mathrm{L}^{2}\left(\mathcal{B}^{0}\right)$, since their $\mathrm{L}^{2}$-norm is not finite. All other representations are infinite-dimensional.

Similarly to Proposition 3.2, we now have

Proposition 4.2. Let $\Gamma$ be a non-uniform lattice in $G$. Then $\Gamma \backslash \mathcal{B}$ is a Ramanujan complex if and only if every irreducible spherical infinite-dimensional representation which is weakly contained in the spectrum of $\mathrm{L}^{2}(\Gamma \backslash G)$ is tempered.

We can now prove:

Theorem 4.3. Assume $d \geq 3$ and $F=\mathbb{F}_{q}((t))$. Then for any congruence subgroup $\Gamma$ of $\mathrm{PGL}_{d}\left(\mathbb{F}_{q}[1 / t]\right), \Gamma \backslash \mathcal{B}$ is not Ramanujan.

Proof. In order to prove that $\Gamma \backslash \mathcal{B}$ is not a Ramanujan complex, we must show that there exists a spherical, infinite-dimensional, irreducible representation occurring in the spectrum of $\mathrm{L}^{2}(\Gamma \backslash G(F))$ (discrete or continuous) which is not tempered. It suffices to treat the case $\Gamma=\mathrm{PGL}_{d}\left(\mathbb{F}_{q}[1 / t]\right)$.

Let $G=\mathrm{PGL}_{d}, k=\mathbb{F}_{q}(t)$ and let $\mathbb{A}$ be the adèles ring of $k$. The group $G(\mathbb{A})$ acts on $\mathrm{L}^{2}(\mathrm{Z}(\mathbb{A}) G(k) \backslash G(\mathbb{A}))$ via the right regular representation $\rho$, where $\mathrm{Z}$ denotes the center. By strong approximation, the irreducible representations in the spectrum of $\mathrm{L}^{2}(\Gamma \backslash G(F))$ are precisely the local components of automorphic representations $\pi$ in $\mathrm{L}^{2}(\mathrm{Z}(\mathbb{A}) G(k) \backslash G(\mathbb{A}))$ that have a $U$-invariant vector, where $U \subseteq G(\mathbb{A})$ is a subgroup such that $G(F) U$ is open and $\Gamma=G(k) \cap G(F) U$ (see [LSV1, Proposition 3.4]).

We apply the theory of Langlands to show that if $d \geq 3$, then there exists an infinite-dimensional irreducible unitary representation $\pi=\bigotimes \pi_{v}$ of $G(\mathbb{A})$ occurring in the spectral decomposition of $\rho$, such that $\pi_{v}$ is spherical and non-tempered for every place $v$ of $k$.

Let $P=M N$ be a parabolic subgroup of $G$ whose Levi factor $M$ is isomorphic to $\mathrm{GL}_{1} \times \mathrm{GL}_{d-1}$ (any parabolic $P$ which is not a Borel subgroup would serve equally well). For $s \in \mathbb{C}$, define a character $\chi_{s}$ of $\mathrm{M}(\mathbb{A})$, by

$$
\chi_{s}\left(g_{1}, g_{2}\right)=\left|g_{1}\right|^{(d-1) s}\left|\operatorname{det}\left(g_{2}\right)\right|^{-s} \text {. }
$$

We may extend $\chi_{s}$ to $P(\mathbb{A})$ by being trivial on $N(\mathbb{A})$ and define

$$
\pi_{s}=\operatorname{Ind}_{P(\mathbb{A})}^{G(\mathbb{A})} \chi_{s}
$$


(using unitary induction). The representations $\pi_{s}=\bigotimes \pi_{s, v}$ are unitary, irreducible, and infinite-dimensional. Since $\mathrm{M}\left(k_{v}\right)$ is not compact modulo its center, $\chi_{s, v}$ is not a tempered representation of $\mathrm{M}\left(k_{v}\right)$. The theory of matrix coefficients shows that $\pi_{s}$ is a non-tempered representation of $G\left(k_{v}\right)$. Furthermore, according to Langlands' theory (see $\left[\mathrm{A}\right.$ ), the direct integral of the representations $\left\{\pi_{s}\right\}$ (relative to Lebesgue measure $d s)$ occurs in the continuous part of $\mathrm{L}^{2}(\mathrm{Z}(\mathbb{A}) G(k) \backslash G(\mathbb{A}))$. This proves the theorem.

Remark 4.4. Since $\mathrm{PGL}_{d}\left(\mathbb{F}_{q}[1 / t]\right)$ is not cocompact, the spectrum of $\mathrm{L}^{2}(\mathrm{Z}(\mathbb{A}) G(k) \backslash G(\mathbb{A}))$ has discrete and continuous components. The proof above shows that $\mathrm{L}^{2}(\Gamma \backslash G(F))$ always has a non-tempered representation in the continuous spectrum. We will show that when $d$ is not a prime and $\Gamma$ is small enough, such representations occur in fact also in the discrete spectrum. Write $d=a b$ where $a, b>1$. Let $P=M N$ be a parabolic subgroup of $G$ whose Levi factor $M$ is isomorphic to $\mathrm{GL}_{a} \times \cdots \times \mathrm{GL}_{a}$ ( $b$ times) modulo the center.

Let $\pi$ be a cuspidal representation of $\mathrm{GL}_{a}(\mathbb{A})$, which acts trivially on the center. Let $\tilde{\pi}$ be the representation of $\mathrm{GL}_{a} \times \cdots \times \mathrm{GL}_{a}$, given by

$$
\tilde{\pi}\left(g_{1}, \ldots, g_{b}\right)=\pi\left(g_{1}\right)\left|\operatorname{det}\left(g_{1}\right)\right|^{(b-1) / 2} \otimes \cdots \otimes \pi\left(g_{b}\right)\left|\operatorname{det}\left(g_{b}\right)\right|^{-(b-1) / 2} .
$$

Note that the determinants $\operatorname{det}\left(g_{j}\right)$ are only defined up to a common scalar, but $\tilde{\pi}$ itself is well-defined. Pull $\tilde{\pi}$ back to $P(\mathbb{A})$ by being trivial on $N(\mathbb{A})$ and set

$$
\Pi=\operatorname{Ind}_{P(\mathbb{A})}^{G(\mathbb{A})} \tilde{\pi} .
$$

Then $\Pi$ has a unique irreducible quotient $\sigma=\otimes \sigma_{v}$ such that $\sigma_{v}$ is spherical in all places where $\pi$ was spherical, and non-tempered for all $v$. Furthermore, $\sigma$ occurs as a discrete summand in $\mathrm{L}^{2}(\mathrm{Z}(\mathbb{A}) G(k) \backslash G(\mathbb{A}))$ ([JS] or $\left.[\mathrm{J}]\right)$.

Note that there are no such cuspidal $\pi$ which are spherical in all $v$, and hence, in general, this construction does not provide non-tempered representations in the discrete spectrum of $\Gamma \backslash G(F)$ when $\Gamma=\mathrm{GL}_{d}\left(\mathbb{F}_{q}[1 / t]\right)$, but for small enough congruence subgroups of $\Gamma, \Pi$ is a non-tempered representation in $\mathrm{L}^{2}(\Gamma \backslash G(F))$.

Remark 4.5. (1) The difference between the cases $d=2$ and $d \geq 3$ can be explained by the fact that in the latter, there exist proper parabolic subgroups other than the Borel subgroup.

(2) In the uniform case, the existence of Ramanujan complexes is proved in [Li]. A different proof, assuming the Jacquet-Langlands correspondence (to transfer between automorphic representations of groups such as $D^{\times} /$Cent, $D$ being a division algebra, and those of $\left.\mathrm{PGL}_{d}(k)\right)$, was given in [LSV1], which was followed by an explicit construction in LSV2.

(3) The construction of non-tempered representations in the proof of Theorem 4.3 fails in the uniform case, because $D^{\times} /$Cent has no non-trivial parabolic subgroups.

\section{Remarks AND SUGgestions FOR FURTHER RESEARCH}

This note shows that the naive analog of Morgenstern's construction does not work for $d>2$. We can still hope to find examples of higher-dimensional nonuniform Ramanujan complexes by using non-uniform arithmetic lattices in $G=$ $\mathrm{PGL}_{d}\left(\mathbb{F}_{q}((t))\right)$, other than $\Gamma=\mathrm{PGL}_{d}\left(\mathbb{F}_{q}[1 / t]\right.$ ) (although for $d=2, \Gamma$ is the only non-uniform lattice, up to commensurability). Other lattices are obtained from 
inner forms of $G$, such as $\mathrm{PGL}_{s}(D)$, where $D$ is a division algebra of degree $d / s$ over a global subfield of $\mathbb{F}_{q}((t))$. If $s>2$, an argument similar to the proof of Theorem 4.3 will show that there are no non-uniform Ramanujan complexes there, but maybe for $s=2$ one may obtain some, as the proof fails in this case.

Yet other lattices are obtained from forms of outer type, namely those obtained from Hermitian forms of degree $s$ over a division algebra of degree $r$, where $r s=$ $d$. Here, an argument similar to the proof of Theorem 1.4 in [LSV1 should also imply that these lattices will not give non-uniform Ramanujan complexes if $s \geq 4$. However, for $d=2 r$ and $\Gamma$ a lattice obtained from a Hermitian form of degree 2 over a division algebra of degree $r$, one may hope to get non-uniform Ramanujan complexes. We don't know what to expect for $d=3 r$.

From a representation-theoretic perspective, one may consider the property of being 'weakly Ramanujan': for $\Gamma$ a lattice in $G(F)$, the quotient $\Gamma \backslash \mathcal{B}$ is weakly Ramanujan if the non-trivial discrete simultaneous spectrum of the $A_{i}$ acting on $\mathrm{L}^{2}(\Gamma \backslash \mathcal{B})$ is contained in the simultaneous spectrum of the $A_{i}$ acting on $\mathrm{L}^{2}(\mathcal{B})$. This has the standard interpretation in terms of representations (compare to Proposition 4.2): $\Gamma \backslash \mathcal{B}$ is weakly Ramanujan if and only if every irreducible spherical infinitedimensional representation which appears in the discrete spectrum of $\mathrm{L}^{2}(\Gamma \backslash G)$ is tempered.

Then we have:

Theorem 5.1. Suppose $d \geq 3$ is prime. Then for every congruence subgroup $\Gamma$ of $\mathrm{PGL}_{d}\left(\mathbb{F}_{q}[1 / t]\right), \Gamma \backslash \mathcal{B}$ is weakly Ramanujan.

On the other hand, when $d$ is composite, there are infinitely many non-uniform quotients of $\mathcal{B}$ which are not weakly Ramanujan.

Since we only deal with the discrete spectrum, the proofs are the same as those given in LSV1, Theorems 1.2,1.3].

It will be very interesting to understand the difference between representations in the discrete and continuous spectrum from a combinatorial point of view.

\section{REFERENCES}

[A] J. Arthur, Eisenstein series and the trace formula, in Automorphic Forms, Representations, and $L$-functions, Proc. Sympos. Pure Math. XXXIII, eds. A. Borel and W. Casselman, Amer. Math. Soc., pp. 253-274, 1979. MR.546601 (81b:10020)

[J] H. Jacquet, On the residual spectrum of GL( $n)$, in Lie group representations, II (College Park, Md., 1982/1983), eds. R. Herb, S. Kudla, R. Lipsman and J. Rosenberg, Lecture Notes in Math. 1041, Springer, pp. 185-208, 1984. MR748508 (85k:22045)

[JS] H. Jacquet and J. Shalika, Sur le spectre résiduel du groupe linéaire [On the residual spectrum of the linear group], C. R. Acad. Sci. Paris Sér. I Math. 293 (11) (1981), 541543. MR647676 (83a:10050)

[La] L. Lafforgue, Chtoucas de Drinfeld et correspondance de Langlands (French), Invent. Math. 147 (1) (2002), 1-241. MR.1875184 (2002m:11039)

[Li] W.-C. W. Li, Ramanujan hypergraphs, Geometric and Functional Analysis 14 (2004), 380-399. MR2060199 (2005i:11172)

[Lu] A. Lubotzky, Discrete Groups, Expanding Graphs and Invariant Measures, Progress in Math. 125, Birkhäuser, 1994. MR.1308046 (96g:22018)

[LPS] A. Lubotzky, R. Philips and P. Sarnak, Ramanujan graphs, Combinatorica 8 (1988), 261-277. MR963118 (89m:05099)

[LSV1] A. Lubotzky, B. Samuels and U. Vishne, Ramanujan complexes of type $\tilde{A}_{d}$, Israel J. Math. 149 (2005), 267-299. MR2191217(2006i:11134)

[LSV2] A. Lubotzky, B. Samuels and U. Vishne, Explicit constructions of Ramanujan complexes of type $\tilde{A}_{d}$, European J. Combinatorics 26 (6) (2005), 965-993. MR2143204 (2006g:20043) 
[MW] C. Mœglin and J.-L. Waldspurger, Le spectre résidual de GL(n), Ann. Scient. École Norm. Sup., $4^{e}$ série 22 (1989), 605-674. MR1026752 (91b:22028)

[M1] M. Morgenstern, Existence and explicit constructions of $q+1$ regular Ramanujan graphs for every prime power q, J. Combin. Theory Ser. B 62 (1) (1994), 44-62. MR1290630 (95h:05089)

[M2] M. Morgenstern, Ramanujan diagrams, SIAM J. Discrete Math. 7 (4) (1994), 560-570. MR.1299084 (96g:05076)

[M3] M. Morgenstern, Natural bounded concentrators, Combinatorica 15 (1) (1995), 111-122. MR.1325275 (96f:05106)

[OW] M.S. Osborne and G. Warner, The Theory of Eisenstein Systems, Academic Press, 1981. MR643242 (83j:10034)

[S] B. Samuels, "Ramanujan complexes, non-uniform quotients and isospectrality", Ph.D. thesis, Yale University, 2005.

[Sa] A. Sarveniazi, Explicit construction of a Ramanujan $\left(n_{1}, n_{2}, \ldots, n_{d-1}\right)$-regular hypergraph, Duke Math. J. 139 (2007), no. 1, 141-171. MR2322678(2008k:11055)

(A. Lubotzky) Institute of Mathematics, Hebrew University, Givat-Ram, Jerusalem 91904, ISRAEL

E-mail address: alexlub@math.huji.ac.il

(U. Vishne) Department of Mathematics, Bar-Ilan University, Ramat-Gan 52900, ISRAEL

E-mail address: vishne@math.biu.ac.il 